\title{
藜个体在高密度种群中的氮素利用效率
}

\author{
袁志友 李凌浩 韩兴国* \\ (中国科学院植物研究所植被数量生态学重点实验室, 北京 100093)
}

\begin{abstract}
摘 要 氮素利用效率 $(N U E)$ 是植物养分策略研究中的一项重要内容。该文利用 Berendse 和 Aerts 提出的氮素利 用效率概念和原理研究了高密度的藜 (Chenopodium album) 种群中不同植物个体在种内竞争条件下的氮素利用效 率。结果表明，由于植株的氮素吸收速率与其个体大小成非线性关系，说明不同植株个体对氮素的竞争属于非对 称竞争。个体较大的植株氮素输入较高, 而个体较小的植株氮素输出较高, 因而较大个体植株的氮素净增加也较 高。植株的氮素损失随着个体大小的增加而增加 较大植株个体的氮素浓度随着生长而下降, 而较小植株个体的 氮素浓度随时间的变化不大, 说明个体较小的植株的生长受光照的限制比受氮素的限制更大, 而对较大的植株个 体而言, 它们的生长受氮素的限制更大。高密度莍种群中的不同植物个体具有不同的养分策略, 氮素利用效率及 其组成部分氮素生产力 $(N P)$ 和氮素滞留时间 (MRT)均不同。植株的 $N P$ 和 $M R T$ 与其个体大小正相关, 较大的植 物个体具有较高的 $N P$ 和较长的 $M R T$, 因而氮素利用效率也高于个体较小的植株。在个体水平上种内不同植株的 $N P$ 与 $M R T$ 不存在权衡关系 (Trade-off)。因此,Berendse 和 Aerts 提出的氮素利用效率概念不仅适用于研究种间的养 分策略，对于研究种内不同植株的养分策略也同样适用。
\end{abstract}

关键词 种内竞争 氮素生产力 滞留时间 氮素利用效率 植物个体大小

\section{NITROGEN USE EFFICIENCY OF COMPETING INDIVIDUALS IN A DENSE STAND OF AN ANNUAL HERB , CHENOPODIUM ALBUM}

\author{
YUAN Zhi-You LI Ling-Hao and HAN Xing-Guo* \\ (Laboratory of Quantitative Vegetation Ecology, Institute of Botany , Chinese Academy of Sciences , Beijing 100093 , China)
}

\begin{abstract}
The concept of nitrogen use efficiency ( $N U E$ ) offers a powerful tool to study plant strategies with respect to nutrient limitation. We studied the $N U E$ of an annual herb, Chenopodium album, in a dense monospecific stand using the concept introduced by Berendse and Aerts. Larger individuals absorbed more $\mathrm{N}$ in greater proportions relative to their size , suggesting that the competition for soil nitrogen was asymmetric (onesided) among individual plants in the stand. Nitrogen loss from individuals also increased with plant size. Nitrogen influx $\left(r_{\text {in }}\right.$, the rate of $\mathrm{N}$ uptake per unit aboveground $\left.\mathrm{N}\right)$ was greater in larger individuals while nitrogen outflux $\left(r_{\text {out }}\right.$, the rate of $\mathrm{N}$ loss per unit aboveground $\left.\mathrm{N}\right)$ was the reverse. Therefore, the relative rate of nitrogen increment $\left(r_{\text {in }}-r_{\text {out }}\right)$ was greater in larger individuals whereas it was around zero in the smallest plants. Larger individuals decreased their $\mathrm{N}$ concentration with time while smaller individuals showed little change in $\mathrm{N}$ concentration. These results suggested that the growth of smaller individuals was limited by light availability rather than by $\mathrm{N}$ availability, and $\mathrm{N}$ limitations were greater in larger individuals. Individual plants in this dense stand of $C$. album differed in their $\mathrm{N}$ economy. $N U E$ and its components, i. e. , MRT and $N P$, were different among individuals in the stand. Both $N P$ and $M R T$ were positively related to plant size. Larger individuals had longer $M R T$ and higher $N P$, both of which contributed to higher $N U E$, than the smaller individuals. No trade-off relationship between $N P$ and $M R T$ was found at the intraspecific level. This study showed that the concept of $N U E$ defined by Berendse and Aerts offered a powerful tool in studing plant strategies within species as well as among species.
\end{abstract}

Key words Intraspecific competition, Nitrogen productivity , Mean residence time, Nitrogen use efficiency , Plant size

植物对光照、水分、养分等资源的吸收和利用特 性是植物生态学研究中的一项重要内容 (Aerts \& Chapin，2000），而反映植物对资源吸收利用特性的
一个主要参数就是资源利用效率 (Aerts \& de Caluwe ，1994)。由于氮素是许多陆地生态系统中的 养分限制因子 (Vitousek \& Howarth, 1991 ; Matson et 
al . , 2002) ,因此大多数养分利用效率方面的研究侧 重于对氮素利用效率 (Nitrogen use efficiency, NUE) 的研究。研究氮素利用效率首先应选择适当的衡量 参数, 但目前尚没有一个统一的参数 (袁志友等, 2003)。Hirose(1971)和 Vitousek (1982)曾将氮素利用 效率定义为植物吸收单位重量的氮素所产生的生物 量 如果系统处于 稳定状态” (Frissel，1981) , 则氮 素利用效率等于植物体内氮素浓度的倒数。 Berendse 和 Aerts (1987) 在 Vitousek (1982)的养分利 用效率概念的基础上, 认为氮素利用效率应由两部 分组成：一是氮素生产力 ( $\mathrm{N}$ productivity, $N P$ ) , 二是 氮素的平均滞留时间 (Mean residence time, MRT) 即 $N U E=N P M R T$ 。

对植物的 $N P 、 M R T$ 和 $N U E$ 的研究, 国外只有 Aerts、Eckstein 等人的工作 (Aerts , 1990 ; Aerts \& de Caluwe , 1994 ; Eckstein \& Karlsson，1997）, 国内目前 还没有涉及到 $N P$ 和 $M R T$ 的报道。上述实验或是 研究亚北极冻原上的湿地植物，或是木本植物，或为 控制性实验，而对草原植物的野外实验研究从未开 展。并且这些研究均是在生态系统水平或群落水平 上进行的,而在个体水平上的养分利用效率研究十 分缺乏。另外,Berendse 和 Aerts (1987) 提出的氮素 利用效率概念是假设系统处于稳定状态，但在个体 水平上, 不同的植株个体在不断地生长和死亡, 而不 是处于稳定状态。因此本文对 Berendse 和 Aerts (1987)提出的氮素利用效率概念进行了一定修正， 以便在个体水平上来研究植物在种内竞争条件下的 氮素利用策略。其基本假设是, 由于个体较大的植 株获取的光照较多, 因而其氮素生产力应比个体较 小的植株要高, 如果氮素生产力与氮素滞留时间之 间存在着权衡关系 (Trade-off) 则个体较小的植株应 具有较长的氮素滞留时间。

在个体水平上研究植物对光照、水分、养分等资 源的竞争利用时，应区分为两种竞争(Weiner et al. , 1997 ; Schwinning \& Weiner,1998):- 是对称竞争 (Symmetric competition) 二是非对称竞争 (Asymmetric competition)。一般认为植物对光线的竞争属于非对 称竞争 (Silvertown \& Lovett-Doust，1993) ,而对养分的 竞争属于对称竞争 (Casper \& Jackson，1997) ,但对于 高密度种群中不同植物个体之间的养分竞争属于哪 种竞争还不清楚, 因此本文的另一个目的是在个体 水平上确定植物对氮素的竞争类型。

\section{1 研究地区自然概况}

实验于 2002 年在内蒙古自治区多伦县进行。
该地区位于锡林郭勒盟南部，地处浑善达克沙地南 缘, 属于典型的农牧交错区。地理范围为 $115^{\circ} 50^{\prime} \sim$ $116^{\circ} 55^{\prime} \mathrm{E} 41^{\circ} 46^{\prime} \sim 42^{\circ} 36^{\prime} \mathrm{N}$ 。低山丘陵地貌, 海拔在 $1150 \sim 1800 \mathrm{~m}$ 之间。属于中温带半干旱大陆性气 候, 年均降水量 $385.5 \mathrm{~mm}$, 蒸发量 $1748 \mathrm{~mm}$ 。年均 气温为 $1.6{ }^{\circ} \mathrm{C}$, 最暖月 (7 月)平均气温为 $18.7{ }^{\circ} \mathrm{C}$, 最 冷月 (1月)平均气温为 $-18.3{ }^{\circ} \mathrm{C}$, 无霜期 $100 \mathrm{~d}$ 左 右, $\geqslant 10{ }^{\circ} \mathrm{C}$ 积温为 $1917.9{ }^{\circ} \mathrm{C}$ (白文明等, 2001)。土 壤主要为栗钙土, 其余依次为风沙土、草甸土和黑钙 土。土地总面积约 $3899.2 \mathrm{~km}^{2}$, 其中耕地、草地和 未利用土地基本上各占 $1 / 3$ 。天然植被以典型草原 为主, 次生林、灌丛和沙地植被也有一定发育。农田 以小麦、荍麦、菾麦等连作种植为主。农田弃耕之 后，一般为一年生杂类草占据，其中藜科一年生草本 植物——藜 (Chenopodium album) 是该地区贫痊生境 中最常见的先锋种类之一。取样地点位于中国科学 院植物研究所多伦恢复生态学试验示范研究站于 2001 年围封的捾草站固定样地内。

\section{2 实验处理与研究方法}

选取一年生草本植物藜为实验材料, 该植物常 出现在扰动环境中, 茎直立, 叶片较大, 便于拟合计 算植株各部分的初始含氮量。在 7 月 4 日, 选择一 块不含其它种类植物的高密度藜样地，建立一个 10 $\mathrm{m} \times 10 \mathrm{~m}$ 的正方形区域,该区域的藜密度为 130 株 $\mathrm{m}^{-2}$,在此区域选择各种高度的个体 33 株， 平均高度为 $54.6 \mathrm{~cm}$, 每株植物都挂塑料标签进行标 记, 并测量高度和叶片数, 测量时要尽量减少对周围 植物的干扰。在该小区内再选择 33 株藜个体, 齐地 面剪下带回实验室。7 月 4 日标记的植株在 7 月 28 日也齐地面收获带回实验室, 这时平均高度已达到 $87.7 \mathrm{~cm}$ 。在实验室内 测量每株植物的高度和叶片 数, 并将每株植物按叶、茎、花分开, 在 $70{ }^{\circ} \mathrm{C}$ 烘干至 少 $48 \mathrm{~h}$ 后称重, 然后粉碎, 过 40 目篮, 用凯氏定氮 法 (Kjeldahl) 测定全氮。植物的个体大小用地上生 物量表示。

藜在 7 月 4 日时的地上生物量及叶、茎、花各部 分的含氮量用拟合方法进行计算 (Hikosaka et al. , 1999 ; Weih ，2001) 其中地上生物量 $(y$, 单位 : g) 用 植株高度 $\times$ 叶片数 $(x$, 单位 $\mathrm{m} \times$ No. $)$ 进行拟合计 算 :

$$
y=6.198 \times 10^{-7} x^{3}-3.349 \times 10^{-4} x^{2}+7.332 \times
$$

$10^{-2} x-0.0033\left(R^{2}=0.997\right)$

叶片含氮量 $(y$, 单位 $\mathrm{mg}$ ) 用植株高度 $\times$ 地上生 
物量 $(x$, 单位 $: m \times g)$ 进行拟合计算:

$y=-0.0754 x^{3}+1.094 x^{2}+15.502 x+5.0513$ $\left(R^{2}=0.999\right)$

茎的含氮量 $(y$, 单位 $: \mathrm{mg})$ 用地上生物量 $(x$, 单 位 : g $)$ 进行拟合计算 :

$$
y=-0.0045 x^{3}-0.1705 x^{2}+17.968 x+0.821
$$

$9\left(R^{2}=0.996\right)$

花的含氮量 $(y$, 单位 $\mathrm{mg})$ 用植株高度 $\times$ 地上生 物量 $(x$, 单位 $: \mathrm{m} \times \mathrm{g})$ 进行拟合计算：

$y=0.0214 x^{3}-0.2569 x^{2}+2.1309 x+0.1208$ $\left(R^{2}=0.997\right)$

由叶、茎、花各部分的含氮量可以得出整株植物 在 7 月 4 日 $\left(t_{1}\right)$ 时的含氮量。

植株在 7 月 28 日 $\left(t_{2}\right)$ 的地上生物量及含氮量 为实测值。

氮损失量 $\left(L_{\mathrm{N}}\right.$, 单位 $\left.: \mathrm{mg}\right)$ 为植株枯叶中的氮含 量, 因为取样期间的降水量较低, 可忽略通过淋洗损 失的氮。这样，从 7 月 4 日到 28 日之间每株植物的 氮吸收速率 $\left(N_{\text {uptake }}\right)$ 和氮损失速率 $\left(N_{\text {loss }}\right)$ 分别为 (Hirose , 1975) :

$$
N_{\text {uptake }}=\frac{N_{2}-N_{1}+L_{N}}{t_{2}-t_{1}}, N_{\text {loss }}=\frac{L_{N}}{t_{2}-t_{1}}
$$

其中 $N_{i}$ 为植株在时间 $t_{i}$ 时的氮含量。

由于在本实验中 植株并不处于稳定状态, 因此 每株植物在 $t_{1}$ 至 $t_{2}$ 时段内的地上平均生物量 $(M)$ 和平均含氮量 $(N)$ 分别用下式表示 (Carlen et al. , 1999 ; Eckstein \& Karlsson , 2001) :

$$
M=\frac{M_{2}-M_{1}}{\ln M_{2}-\ln M_{1}}, N=\frac{N_{2}-N_{1}}{\ln N_{2}-\ln N_{1}}
$$

其中 $M_{i}$ 为植株在时间 $t_{i}$ 时的地上生物量。

氮的周转速率分别用氮素输入速率 $\left(r_{\text {in }}\right)$ 和输 出速率 $\left(r_{\text {out }}\right)$ 来表示 (Hirose, 1971)：

$$
r_{\text {in }}=\frac{N_{\text {uptake }}}{N}, r_{\text {out }}=\frac{N_{\text {loss }}}{N}
$$

由于 Berendse 和 Aerts (1987) 提出的氮素利用 效率概念是假设系统处于稳定状态，但本实验中的 植株不处于稳定状态，因此每株植物的氮素生产力 根据 Vázquez de Aldana 和 Berendse (1997)、Carlen 等 (1999)、Eckstein 和 Karlsson (2001) 给出的公式进行 计算 :

$$
N P=\frac{M_{2}-M_{1}}{t_{2}-t_{1}} \frac{\ln N_{2}-\ln N_{1}}{N_{2}-N_{1}}=\frac{M_{2}-M_{1}}{\left(t_{2}-t_{1}\right) N}
$$

如果 $N_{\text {loss }}=N_{\text {uptake }}$ ，即 $N_{1}=N_{2}$ 时，植株中氮素 处于稳态，如果 $N_{\text {loss }} \neq N_{\text {uptake }}$ 则植株中氮素不处于 稳态, 这时每株植物中的氮素平均滞留时间为：

$$
M R T=\frac{N}{N_{\text {loss }}}=\frac{1}{r_{\text {out }}}
$$

由于氮素利用效率为氮素生产力与氮素平均滞 留时间的乘积 (Berendse \& Aerts , 1987), 所以, 每株 植物的氮素利用效率为：

$N U E=N P \quad M R T=\frac{\mathrm{M}_{2}-M_{1}}{\left(t_{2}-t_{1}\right) N} \frac{N}{N_{\text {loss }}}=\frac{M_{2}-M_{1}}{L_{N}}$

采用 SSPS10.0 软件进行统计分析。

\section{3 结果与分析}

藜不同个体在两次收获时的氮素浓度如图 1 所 示。可以看出, 在 7 月 4 日(图 1a)和 28 日(图 1b)两 次测定中, 均表现为个体较大的植株的氮素浓度低 于个体较小的植株。植株氮素浓度与植株个体大小 呈负相关，相关关系分别为: $y=36.635 x^{-0.1436}\left(R^{2}\right.$ $=0.4898) 、 y=32.777 x^{-0.1008}\left(R^{2}=0.3610\right)$ 。在 7 月 4 日, 全部植株的平均氮素浓度为 $38.41 \mathrm{mg} \mathrm{g}^{-1}$, 而在 7 月 28 日的平均氮素浓度为 $28.53 \mathrm{mg} \mathrm{g}^{-1}$, 说 明植株氮素浓度随着生物量的积累而下降。

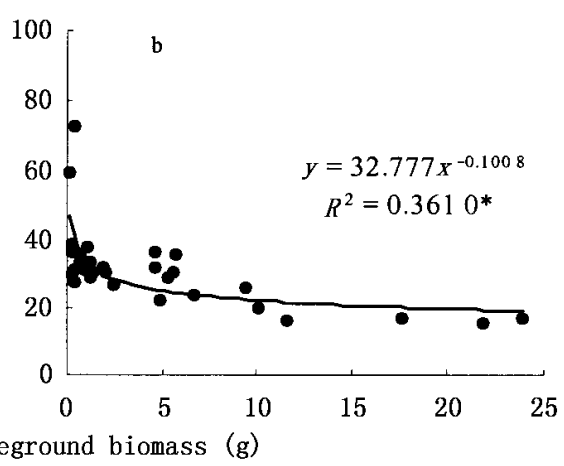

图 1 不同植株个体在不同收获时期 ( a : 7 月 4 日 ; b : 7 月 28 日)的氮素浓度

Fig. $1 \mathrm{~N}$ concentrations of individual plants at two harvesting periods (a : July 4 ; b : July 28) 
植株在整个测定期间，平均含氮量、氮素吸收速 率和氮素损失速率均随着植株个体大小的增长而增 加(图 2)。个体较大的植株的含氮量也较高 (图 2a) 氮素吸收速率与其个体大小呈幂函数关系 (图 $2 b$ ），说明植株对氮素的竞争属于非对称竞争，同样， 个体较大的植株的氮素损失速率也较高 (图 2c) ,氮 素吸收速率和氮素损失速率并不相等, 说明植株中 的氮素不处于稳定状态。

植株的氮素周转速率也随着植株个体大小的变 化而变化(图 3) , 其中, 氮素的输入随着植株个体生 物量的增加而增加 (图 3a), 但氮素输出与之相反 (图 3b) ,由于氮素输入大于氮素输出,氮素的净增 加也随着植株个体大小的增加而增加 (图 3c)。

根据 Berendse 和 Aerts (1987)提出的氮素利用 效率概念，在个体水平上对藜不同个体的氮素生产 力 $(N P)$ 、氮素滞留时间 $(M R T)$ 和氮素利用效率 $(N U E)$ 进行了计算。结果表明，在种内竞争条件下 植株个体具有不同的氮素利用策略 (图 4)。氮素生 产力随着植株个体的增加呈上升趋势 (图 4a) ,氮素 的平均滞留时间与之类似 (图 4b) ,由于氮素利用效 率为氮素生产力与氮素平均滞留时间的乘积, 因而
氮素利用效率也随着植株个体大小的增加而增加 （图 4c），即个体越大，氮素利用效率越高，但氮素生 产力和氮素平均滞留时间之间不存在负相关性 (图 4d）,因而其间未表现出权衡关系。

\section{4 讨 论}

利用 Berendse 和 Aerts (1987) 提出的氮素利用 效率概念对高密度藜种群中不同植物个体在种内竞 争条件下的氮素利用效率进行了研究 结果表明, 不 同的植株个体在种内竞争条件下表现出不同的氮素 利用策略。植株的含氮浓度与植株个体大小呈幂函 数关系(图 1), 被遮荫的较小植株个体的含氮浓度 较高, 说明植物对氮素的吸收不仅受土壤可利用氮 素的影响，还受植株自身氮素状况的影响，即植物对 氮素的吸收受供求关系的双重影响。Anten 和 Werger (1996)也发现遮荫植物的氮素浓度较高。从 7 月 4 日到 28 日，个体较大的植株氮素浓度下降得 更多(图 1)，说明较大植株个体的生长受氮素的限 制更大，而对个体较小的植株来说，它们的氮素浓度 变化不大 ,光对其生长的限制作用比氮素更大。个 体较大的植株对氮素的吸收速率和氮素损失速率
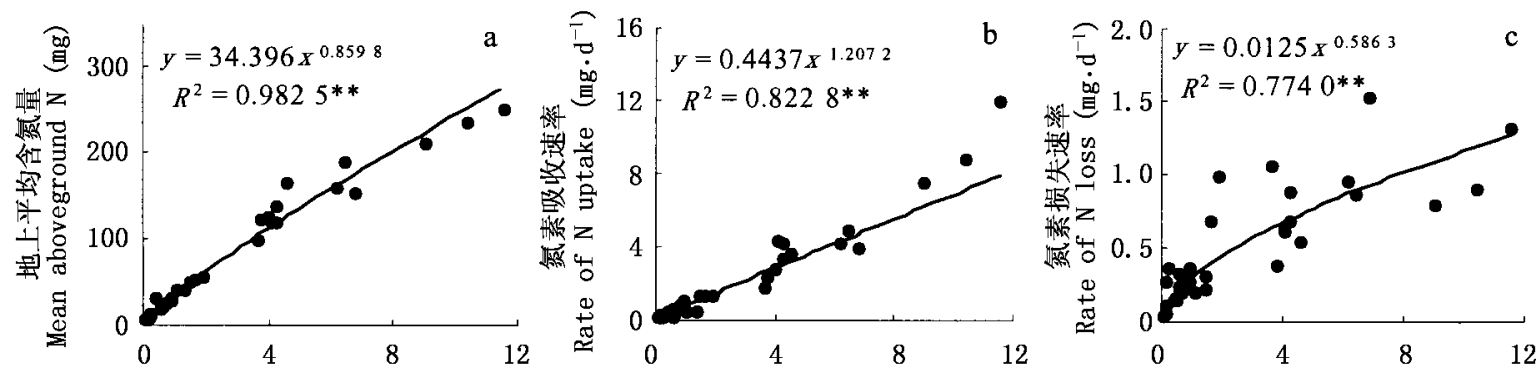

图 2 不同植株个体的地上平均含氮量 (a)、氮素吸收速率 (b) 和氮素损失速率 (c)

Fig.2 The relationships between the mean aboveground $\mathrm{N}$ content (a), rate of $\mathrm{N}$ uptake (b) , rate of $\mathrm{N}$ loss (c) and the mean aboveground biomass of each individual
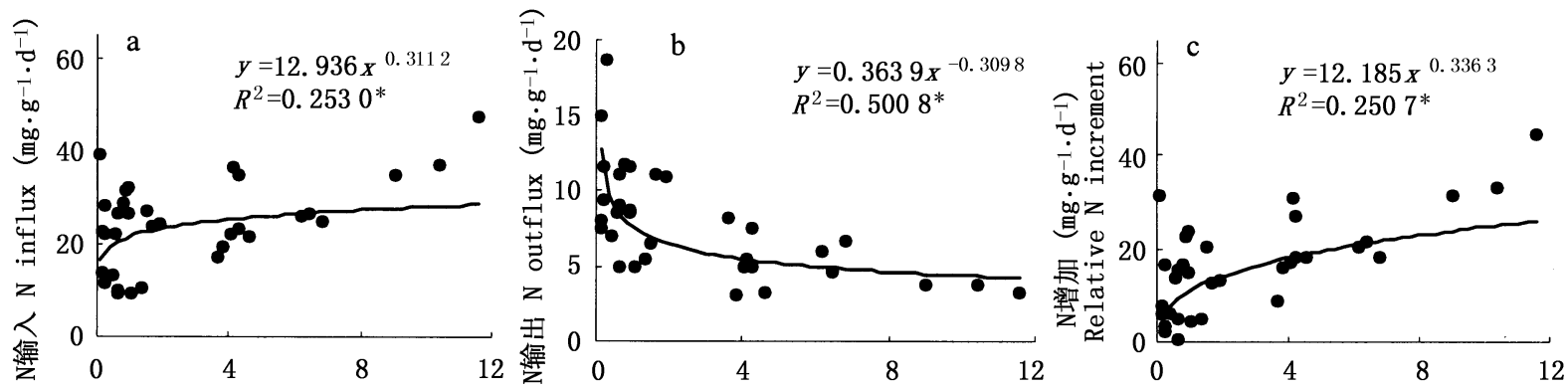

地上生物量 Mean aboveground biomass（g)

图 3 不同植株个体的 (a) $\mathrm{N}$ 输入、 (b) $\mathrm{N}$ 输出、 (c) N 净增加

Fig.3 The relationships between (a) the $\mathrm{N}$ influx $\left(r_{\text {in }}\right),\left(\right.$ b) the $\mathrm{N}$ outflux $\left(r_{\text {out }}\right),(\mathrm{c})$ the relative rate of net $\mathrm{N}$ increment rate

$\left(r_{\text {in }}-r_{\text {out }}\right)$ and the mean aboveground biomass of each individual 


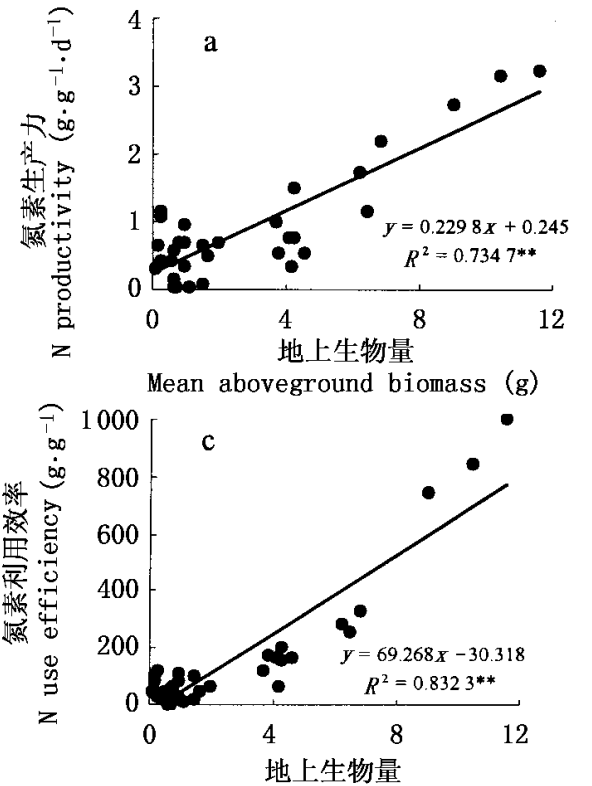

Mean aboveground biomass $(\mathrm{g})$

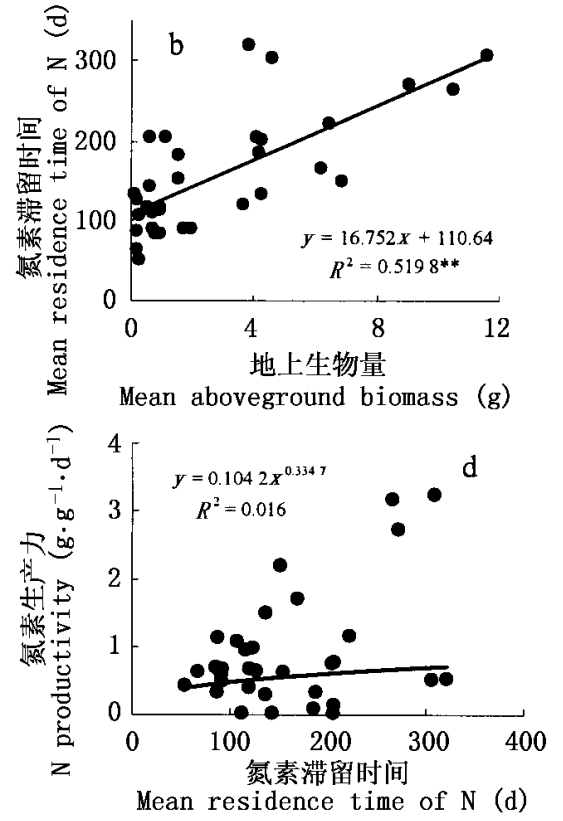

图 4 不同植株个体的 (a)氮素生产力、 (b)氮素滞留时间、(c)氮素利用效率、(d)氮素生产力与氮素滞留时间之间的关系 Fig.4 (a) the $\mathrm{N}$ productivity, (b) the mean residence time, (c) the $\mathrm{N}$ use efficiency, (d) the relationship between $\mathrm{N}$ productivity $(N P)$ and mean residence time $(M R T)$

都高于个体较小的植株 (图 2), 但由于个体较大的 植株具有较高的氮素输入和氮素输出, 因而氮素的 净增加量也较高 (图 3) ,并且较大的植株个体具有 较高的 $N P$ 和 $M R T$, 既然 $N U E$ 为 $N P$ 与 $M R T$ 的乘 积, 因而较大的植株个体也具有较高的 $N U E$ (图 4)。

如果 $N P$ 与 $M R T$ 之间存在权衡关系 (Eckstein et $a l$ ，1999）则植物要提高 $N U E$, 有两种途径 :或者 提高 $N P$ 或者提高 $M R T$ 。个体较小的植株由于 $N P$ 较低（图 4a）,因此要提高 $N U E$ 就必须提高其 $M R T$ 。 但在本实验中，个体较小的植株的 $M R T$ 却较小 (图 $4 \mathrm{~b})$, 说明在种内, $N P$ 与 $M R T$ 之间并不存在权衡关 系。而在种间, $N P$ 与 $M R T$ 之间存在权衡关系 (Aerts , 1990 ; Eckstein et al. , 1999)。NP 和 MRT 之 间在种内和种间表现为不同的关系可能有以下原因 (袁志友等，2003）:1) NP、MRT 及相关特性在种内的 变化比在种间的变化要小(Aerts \& de Caluwe, 1994 ; Eckstein et al. , 1999) , 从而造成数据中的干执 噪 声”过高 2) NP 和 MRT 的关系可能还受其它相关因 素调控(Aerts , 1990 ; Garnier \& Aronson , 1998 ; Eckstein et al. ,1999）3）由于氮库的计算是用 $N P$ 作分 母、MRT 作分子来衡量的，因而存在自相关性问题。

植物对光照、水分、养分等资源的对称竞争指竞 争能力与植株个体大小成正比, 而非对称竞争指植 株获取的资源与其个体大小不成线性关系 (Schwinning \& Weiner , 1998)。一般认为植物对光照的竞争 属于非对称竞争 (Weiner, 1990 ; Anten \& Hirose ,
1998 ; Hikosaka et al，，1999），而对养分的竞争属于 对称竞争(Weiner et al. , 1997)。但在该研究中，植 株的氮素吸收速率与其个体大小成非线性关系 (图 $2 b ）$,说明在种内竞争条件下，高密度种群中的植物 个体对氮素的吸收属于非对称竞争, 这可能与植株 个体对光照的非对称竞争有关，因为较小的植株个 体处于下层，对氮素的需求会较低。

本研究没有测定根系对氮素的吸收利用, 虽然 Aerts (1990) 曾发现瑞典北部多年生植物彩营石楠 (Calluna vulgaris)、酸沼草 (Molinia caerulea) 的根系 对氮素的吸收利用有较大的影响, 但本实验中的葱 属于一年生草本植物，它不象多年生植物那样有具 有咜藏功能的根系 根系对 NUE 及其组成部分的影 响可能相对要小, 因而可以忽略不计。

氮素利用效率是植物利用限制性养分策略研究 中的一个重要概念, 可应用于从叶片到生态系统等 各种尺度。人们对叶片 (Field \& Mooney, 1986 ; Evans , 1989)、植株 (Hirose , 1975 ; Berendse \& Aerts , 1987) 和群落 (Bridgham et al. , 1995 ; Hiremath \& Ewel，2001) 尺度上的 NUE 作了大量研究, 但涉及到 氮素生产力和氮素滞留时间的研究很少, 并且还有 许多问题有待解决, 如氮素利用效率在不同尺度之 间的联系，水分、光线等资源互作对氮素利用效率的 影响，以及各种养分之间的吸收和耦合关系等。虽 然大多数情况下, 氮素利用效率高的物种具有更强 的竞争优势，但也存在例外的情况（Chapin，1980)， 
对于生长于肥沃土壤上的杂草和具有䛎藏养分功能 的植物来说, 如果用植物吸收单位养分所产生的生 物量来计算氮素利用效率,则这些植物的氮素利用 效率都较低，因此应进一步确定合适的参数来准确 计算氮素利用效率。目前涉及氮素生产力和氮素滞 留时间的氮素利用效率研究还很少, 国内尚没有这 方面的报道 特别是在叶片、植株和群落等各种尺度 上的有关研究有待加强。

\section{参 考 文 献}

Aerts, R. 1990. Nutrient use efficiency in evergreens and species from heathlands. Oecologia, 84: $391 \sim 397$.

Aerts, R. \& F.S. Chapin. III. 2000. The mineral nutrition of wild plants revisited: a re-evaluation of processes and patterns. Advances in Ecological Research, 30: 1 67 .

Aerts, R. \& H. de Caluwe. 1994. Nitrogen use efficiency of Carex species in relation to nitrogen supply. Ecology, 75: 2362 $\sim 2372$.

Anten, N.P.R. \& T. Hirose. 1998. Biomass allocation and light partitioning among dominant and subordinate individuals in Xanthium canadense stands. Annals of Botany, 82: $665 \sim 673$.

Anten, N.P.R. \& M.J.A. Werger. 1996. Canopy structure and nitrogen distribution in dominant and subordinate plants in a dense stand of Amaranthus dubius L. with a size hierarchy of individuals. Oecologia, 105: $30 \sim 37$.

Bai, W. M. (白文明), L. H. Li (李凌浩) \& S. H. Song (宋世 环). 2001. Analyses on the status quo of resources utilization in Duolun County of Inner Mongolia. Journal of Arid Land Resources and Environment (干旱区资源与环境), 15: 63 67. (in Chinese with English abstract)

Berendse, F. \& R. Aerts. 1987. Nitrogen-use-efficiency: a biological meaningful definition? Functional Ecology, 1: $293 \sim 296$.

Bridgham, S.D. , J. Pastor, C.A. McClaugherty \& C.J. Richardson. 1995. Nutrient-use efficiency: a litterfall index, a model, and a test along a nutrient availability gradient in North Carolina peatlands. American Naturalist, 145: $1 \sim 21$.

Carlen, C. , R. Kölliker \& J. Nösberger. 1999. Dry matter allocation and nitrogen productivity explain growth responses to photoperiod and temperature in forage grasses. Oecologia, 121: 441 $\sim 446$.

Casper, B. B. \& R. B. Jackson. 1997. Plant competition underground. Annual Review of Ecology and Systematics, 28: 545 570 .

Chapin, III. F.S. 1980. The mineral nutrition of wild plants. Annual Review of Ecology and Systematics, 11: $233 \sim 260$.

Eckstein, R.L. \& P.S. Karlsson. 1997. Above-ground growth and nutrient use by plants in a subarctic environment: effects of habitat, life-form and species. Oikos, 79:311 324 .

Eckstein, R.L. \& P.S. Karlsson. 2001. Variation in nitrogen-use efficiency among and within subarctic graminoids and herbs. New Phytologist, 150: $641 \sim 651$.

Eckstein, R.L., P.S. Karlsson \& M. Weih. 1999. Leaf life span and nutrient resorption as determinants of plant nutrient conserva- tion in temperate-arctic regions. New Phytologist, 143: 177 189.

Evans, J. R. 1989. Photosynthesis and nitrogen relationships in leaves of $\mathrm{C}_{3}$ plants. Oecologia, 78: $9 \sim 19$.

Field, C. B. \& H.A. Mooney. 1986. The photosynthesis-nitrogen relationship in wild plants. In: Givinish, T.J. ed. On the economy of plant form and function. Cambridge: Cambridge University Press. $25 \sim 56$.

Frissel, M.J. 1981. The definition of residence times in ecological models. Ecological Bulletins, 33: $117 \sim 122$.

Garnier, E. \& J. Aronson. 1998. Nitrogen-use efficiency from leaf to stand level: clarifying the concept. In: Lambers, H., H. Poorter \& M. M. U. van Vuuren eds. Inherent variation in plant growth. Physiological mechanisms and ecological consequences. Leiden (The Netherlands) : Backhuys Publishers. $515 \sim 538$.

Hikosaka, K. , S. Sudoh \& T. Hirose. 1999. Light acquisition and use of individuals competing in a dense stand of an annual herb Xanthium canadense. Oecologia, 118: $388 \sim 396$.

Hiremath, A.J. \& J. J. Ewel. 2001. Ecosystem nutrient use efficiency, productivity, and nutrient accrual in model tropical communities. Ecosystems, 4: 669 682 .

Hirose, T. 1971. Nitrogen turnover and dry matter production of a Solidago altissima population. Japanese Journal of Ecology, 21 : $18 \sim 32$.

Hirose, T. 1975. Relations between turnover rate, resource utility and structure of some plant populations: a study in the matter budgets. Journal of the Faculty of Science, The University of Tokyo, 11: $335 \sim 407$.

Matson, P. A., K. Lohse \& S. Hall. 2002. The globalization of nitrogen deposition: consequences for terrestrial ecosystems. Ambio, 31: $113 \sim 119$.

Schwinning, S. \& J. Weiner. 1998. Mechanisms determining the degree of size-asymmetry in competition among plants. Oecologia, 113: $447 \sim 455$.

Silvertown, J. W. \& J. Lovett-Doust. 1993. Introduction to plant population biology. 3rd ed. London: Blackwell Scientific. 210.

Vázquez de Aldana, B.R. \& F. Berendse. 1997. Nitrogen-use-efficiency in six perennial grasses from contrasting habitats. Functional Ecology, 11: 619 626.

Vitousek, P.M. 1982. Nutrient cycling and nitrogen use efficiency. American Naturalist, 119: $553 \sim 572$.

Vitousek, P. M. \& R.W. Howarth. 1991. Nitrogen limitation on land and in the sea: how can it occur? Biogeochemistry, 13: 87 $\sim 115$.

Weih, M. 2001. Evidence for increased sensitivity to nutrient and water stress in a fast-growing hybrid willow compared with a natural willow clone. Tree Physiology, 21: 1141 1148 .

Weiner, J. 1990. Asymmetric competition in plant populations. Trends in Ecology and Evolution, 5: $360 \sim 364$.

Weiner, J., D. B. Wright \& S. Castro. 1997. Symmetry of belowground competition between Kochia scoparia individuals. Oikos, 79: $85 \sim 91$.

Yuan, Z.Y.(袁志友), L.H. Li(李凌浩) \& J.H. Huang(黄建 辉). 2003. On plant nutrient use efficiency (1) some aspects in reassessing plant nutrient use efficiency. In: Li, C.S. (李承森) ed. Advances in plant sciences. Bejing: China Higher Education Press. $187 \sim 200$. (in Chinese) 